
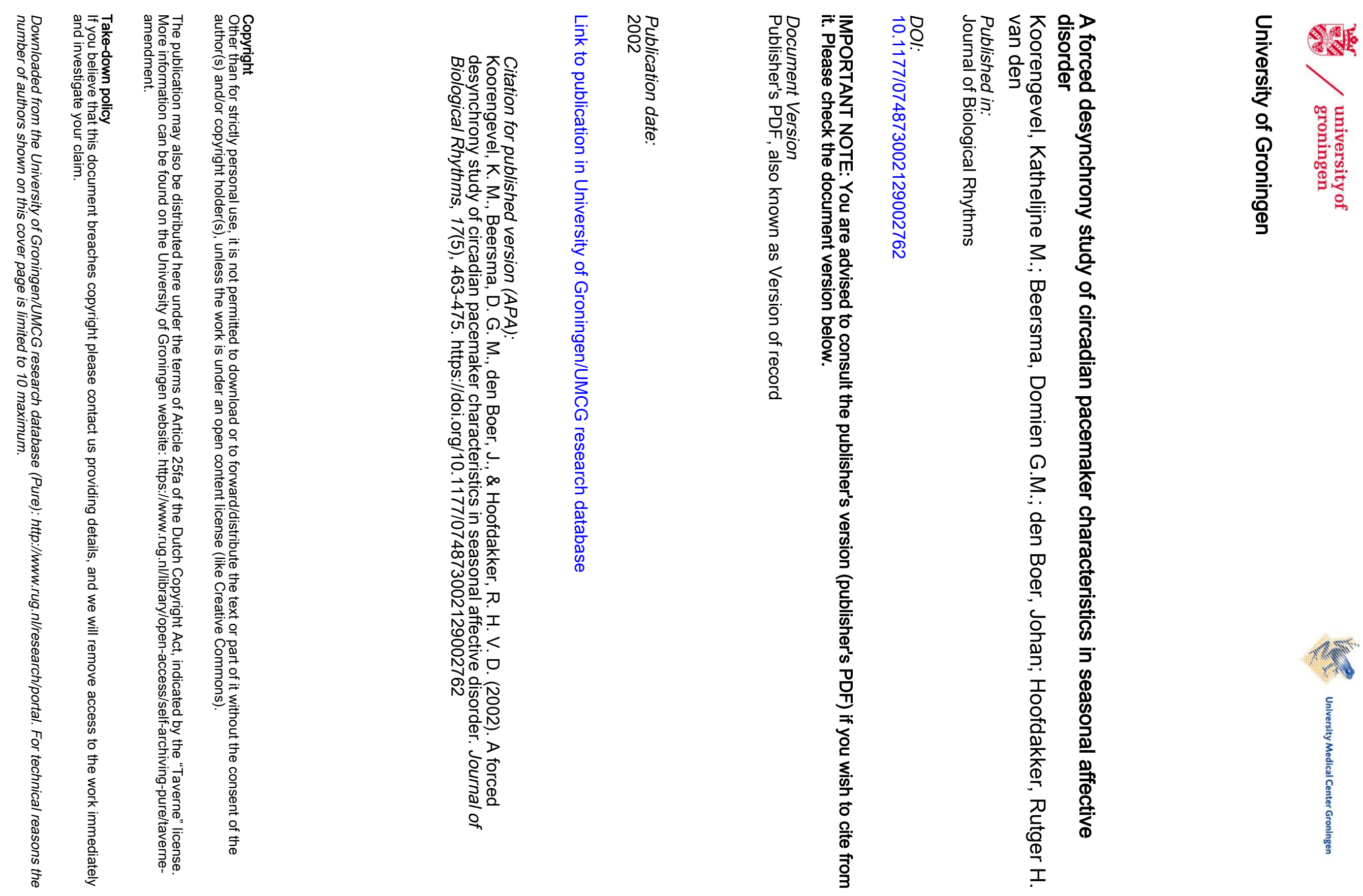


\title{
A Forced Desynchrony Study of Circadian Pacemaker Characteristics in Seasonal Affective Disorder
}

\author{
Kathelijne M. Koorengevel, ${ }^{*}$ Domien G. M. Beersma, ${ }^{*,+, 1}$ \\ Johan A. den Boer,* and Rutger H. van den Hoofdakker* \\ *Department of Psychiatry, University of Groningen, Groningen, the Netherlands, \\ ${ }^{+}$Zoological Laboratory, University of Groningen, Groningen, the Netherlands
}

\begin{abstract}
The circadian pacemaker is an endogenous clock that regulates oscillations in most physiological and psychological processes with a near 24-h period. In many species, this pacemaker triggers seasonal changes in behavior. The seasonality of symptoms and the efficacy of light therapy suggest involvement of the circadian pacemaker in seasonal affective disorder (SAD), winter type. In this study, circadian pacemaker characteristics of SAD patients were compared with those of controls. Seven SAD patients and matched controls were subjected to a 120-h forced desynchrony protocol, in which core body temperature and melatonin secretion profiles were measured for the characterization of circadian pacemaker parameters. During this protocol, which enables the study of unmasked circadian pacemaker characteristics, subjects were exposed to six 20-h days in time isolation. Patients participated twice in winter (while depressed and while remitted after light therapy) and once in summer. Controls participated once in winter and once in summer. Between the SAD patients and controls, no significant differences were observed in the melatonin-derived period or in the phase of the endogenous circadian temperature rhythm. The amplitude of this rhythm was significantly smaller in depressed and remitted SAD patients than in controls. No abnormalities of the circadian pacemaker were observed in SAD patients. A disturbance in thermoregulatory processes might explain the smaller circadian temperature amplitude in SAD patients during winter.
\end{abstract}

Key words seasonal affective disorder, circadian pacemaker, circadian rhythms, core body temperature, melatonin, forced desynchrony

The remarkable seasonal fluctuation of mood and the efficacy of bright-light therapy in patients with seasonal affective disorder (SAD), winter type, have suggested involvement of the circadian pacemaker in the pathogenesis of this disorder. The present study is devoted to the search for possible circadian abnormalities in SAD patients.
The circadian pacemaker is localized in the suprachiasmatic nucleus (SCN) of the brain and operates as an endogenous timekeeping mechanism (Van Esseveldt et al., 2000). This pacemaker generates oscillations in many physiological and psychological processes (Aschoff, 1965) with a near 24-h period (Hiddinga et al., 1997; Carskadon et al., 1999; Czeisler

1. To whom all correspondence should be addressed: Zoological Laboratory, University of Groningen, P.O. Box 14, 9750 AA Haren, the Netherlands; e-mail: d.g.m.beersma@biol.rug.nl.

JOURNAL OF BIOLOGICAL RHYTHMS, Vol. 17 No. 5, October 2002 463-475

DOI: $10.1177 / 074873002237140$

(C) 2002 Sage Publications 
et al., 1999). By its sensitivity to light, the circadian pacemaker is able to respond to seasonal changes in day length, or photoperiod. Throughout the day, retinal light exposure synchronizes this biological clock with the environmental light:dark cycle (Honma and Honma, 1988; Minors et al., 1991; Boivin et al., 1996; Jewett et al., 1997).

In human circadian pacemaker research, the variations of body temperature and of melatonin secretion are often used to study pacemaker output. However, despite the relative ease with which these variables can be measured, the interpretation of these measures in terms of circadian output is complicated by several masking factors. For instance, the nightly secretion of melatonin, which is dependent on the timing and duration of the photoperiod (Lewy et al., 1985), can be directly suppressed by light exposure (Lewy et al., 1980). Therefore, the most accurate estimates of circadian pacemaker characteristics derived from the melatonin secretion profile are obtained in dim light. The endogenous circadian variation of body temperature, in turn, is modulated by the effects of activity, meals, and sleep. The circadian component in temperature data can be obtained by experimentally controlling for masking factors in a constant routine-or a forced desynchrony (FD) protocol. In a constant routine procedure, masking factors are kept constant by keeping subjects in a supine posture and feeding them frequent small meals and exposing them to a prolonged period of wakefulness (Mills et al., 1978; Czeisler et al., 1985). However, the sleep deprivation induced by the constant routine procedure elicits a worsening of mood in healthy subjects (Brendel et al., 1990) and an improvement of mood in depressed patients (Graw et al., 1998). Therefore, this procedure has limitations for studies in which mood regulation is examined. The FD protocol is more suitable because it enables control over the relevant masking factors without eliminating them, while sleep deprivation effects are avoided. During FD, participants are subjected to a sleep-wake cycle that is shorter or longer than $24 \mathrm{~h}$ (i.e., either $20 \mathrm{~h}$ or $28 \mathrm{~h}$ ). The subjective days are spent in dim light ( $<10$ lux) and have a fixed temporal structure. The circadian pacemaker is not able to adapt to this unusual alternation of wakefulness and sleep and starts to oscillate with its own endogenous period. Consequently, the pacemaker and the sleepwake cycle desynchronize (Kleitman and Kleitman, 1953; Czeisler et al., 1986; Dijk et al., 1992). Assuming that circadian and masking factors contribute additively to the overt rhythms measured, a mathe- matical procedure enables the distinction between these two contributors to the measurements (Hiddinga et al., 1997; Czeisler et al., 1999) without the need for prolonged sleep deprivation. Therefore, the method of FD was used in the present study.

\section{CIRCADIAN RHYTHM STUDIES IN SAD}

The hypotheses concerning the involvement of the circadian pacemaker in SAD have mainly focused on circadian phase and amplitude. It has been postulated that in SAD, the circadian pacemaker is phase-shifted relative to the timing of the habitual sleep-wake cycle (in most patients, the pacemaker is phase-delayed) and that the phase-advancing properties of morning light account for the efficacy of light treatment (Lewy, Sack, Singer, et al., 1987). Alternatively, a diminished circadian amplitude has been hypothesized to be involved in the pathogenesis of SAD. According to this hypothesis, amplitude-enhancing effects of light applied in daytime might explain the beneficial effects of light treatment in SAD (Czeisler et al., 1987).

The majority of studies addressing the circadian hypotheses in SAD are naturalistic (i.e., carried out under normal sleep timing), which means that masking effects could be involved. Therefore, the results of these studies at times provide limited information about the circadian pacemaker. A few studies addressed the issue with a constant routine protocol.

\section{NATURALISTIC STUDIES IN SAD}

Several studies have been published in which the melatonin secretion patterns of SAD patients in dim light are compared to those found in controls. Melatonin secretion patterns are studied because they are largely controlled by the circadian pacemaker and are relatively insensitive to most kinds of masking influences. Some of these revealed a delay in dim-light melatonin onset (DLMO) (i.e., time at which a criterion level of melatonin concentration is reached) in SAD patients (Lewy, Sack, Miller, et al., 1987; Sack et al., 1990; Lewy et al., 1998), whereas others did not (Checkley et al., 1993; Thompson et al., 1997). Phaseadvance shifts of DLMO have been reported after morning-light therapy and phase-delay shifts after evening-light therapy in both patients (Lewy, Sack, Miller, et al., 1987; Lewy et al., 1998; Terman et al., 2001) and controls (Lewy, Sack, Miller, et al., 1987; 
Lewy et al., 1998). However, in two studies a phase advance of DLMO after morning-light therapy was observed in patients only (Sack et al., 1990; Thompson et al., 1997). Studies in which entire melatonin secretion profiles were reported demonstrated similar amounts of melatonin production and amplitudes in patients before (Checkley et al., 1993; Thompson et al., 1997) and after (Thompson et al., 1997) light therapy. Likewise, a similar total melatonin production was found in patients before and after morning- or eveninglight therapy (Terman et al., 2001). Comparisons of temperature data have demonstrated no differences in circadian temperature parameters between SAD patients and controls in both winter (Rosenthal et al., 1990; Eastman et al., 1993; Schwartz et al., 1997) and summer (Levendosky et al., 1991; Schwartz et al., 1997). The mean level of body temperature was significantly lower in summer than in winter in both patients and controls in one of these studies (Levendosky et al., 1991), whereas another study revealed a significant lowering of the nocturnal body temperature in summer in patients only (Schwartz et al., 1997). Following light therapy, an increase in amplitude of the circadian temperature profile has been found when patients received a combination of morning and evening light (Rosenthal et al., 1990). However, in a morning-light therapy study, it was observed that the circadian temperature amplitude of patients and controls remained unaffected (Eastman et al., 1993). Both groups showed similar phaseshifting responses.

\section{CONSTANT ROUTINE STUDIES IN SAD}

In the two constant routine studies done so far, lasting 27 and $40 \mathrm{~h}$, respectively, DLMO and the circadian rhythms in core body temperature were measured in female SAD patients and controls (Dahl et al., 1993; Wirz-Justice et al., 1995). From all investigated circadian parameters in the 40-h study, only the midrange crossing of the rising morning limb of the temperature curve showed a delayed phase position of patients relative to controls. Midday light therapy did not change salivary melatonin parameters but resulted in phase advances of some aspects of the temperature curves in patients only (Wirz-Justice et al., 1995). In the 27-h constant routine study, DLMO and the circadian rhythm in core body temperature were measured (Dahl et al., 1993). DLMO and the circadian temperature minimum were significantly phase-delayed in patients in comparison to those in controls. Patients showed an advance after morning-light therapy. In both studies, no differences in the amplitude of the melatonin secretion patterns and of the body temperature curves were observed between patients and controls, nor did the amplitudes change significantly in response to treatment. Constant routine protocols have the disadvantage that they entail sleep deprivation and therefore potentially yield a change of mood. Indeed, visual analogue scale data on mood revealed an antidepressant effect during the 40-h constant routine study (Wirz-Justice et al., 1995). No changes of mood were found in the 27-h constant routine experiment, but after finishing the protocol, some subjects experienced improvement (Avery et al., 1997).

In conclusion, the data do not yield a consistent picture of the circadian system in SAD patients. The reasons for the lack of consistency probably are small sample sizes and that masking factors could not be avoided in those studies. An alternative way to examine the circadian system is to apply an FD protocol. To the best of our knowledge, the present study is the first in which this was done in SAD patients.

\section{METHODS AND MATERIALS}

\section{Recruitment and Selection Criteria}

SAD patients of our outpatient clinic, who were known to be good responders to light therapy in previous years, received written general information on the study. Healthy controls were recruited by advertisements in local newspapers and on local television. Subjects who were interested first received a detailed description of the study by letter and were then invited for a screening procedure if they considered participation. Patients were diagnosed according to the DSM-IV criteria for recurrent major depression with seasonal pattern (American Psychiatric Association, 1994) and had to meet the Rosenthal criteria for SAD (Rosenthal et al., 1984). Controls had to have no psychological complaints or sleeping problems. To evaluate general psychological health, depressed mood, seasonality, and morningness/eveningness, both patients and healthy subjects completed a number of questionnaires: the Beck Depression Inventory (BDI) (Beck et al., 1979); the Structured Interview Guide for the Hamilton Depression Rating ScaleSeasonal Affective Disorder-Self-Rating version (SIGH-SAD-SR) (Williams et al., 1992), consisting of 
the 21-item Hamilton Depression Rating Scale (HDRS) and an 8-item Atypical Symptom Scale (ATYP); the General Health Questionnaire (GHQ) (Goldberg and Williams, 1988); the Seasonal Personality Assessment Questionnaire (SPAQ) (Rosenthal et al., 1987); and the Morningness-Eveningness (M-E) questionnaire (Horne and Ostberg, 1976). Criteria for participation as a control subject were a BDI score $<9$, a SIGH-SAD-SR score $<8$, a GHQ score $<3$, and a SPAQ score $<8$ (Kasper et al., 1989). All subjects were examined by a physician and were declared physically fit. In women, the menstrual cycle was assessed through self-report. With the exception of oral contraceptive pills and nonsteroidal anti-inflammatory drugs (NSAIDs), neither patients nor controls used any medication at least 1 month prior to the experiment. Nobody was dependent on alcohol or caffeine. During 6 months before the study, SAD patients did not use psychoactive drugs. After a verbal explanation of the main characteristics of the protocol, subjects gave written informed consent. The study was approved by the Medical Ethics Committee of the Groningen Academic Hospital. Patients and controls were matched for age, sex, phase of the menstrual cycle, and smoking habits. SAD patients were engaged in the study once during a depressive episode, once when remitted after light therapy, and once in summer. Controls participated once in winter and once in summer. Both patients and controls were paid for their participation.

During the winter season, BDI and SIGH-SAD-SR ratings were obtained weekly from SAD patients. Participation during a depressive episode required a BDI score $\geq 16$. Light therapy thereafter consisted of $45 \mathrm{~min}$ of bright light once a day with an intensity of 10,000 lux for 5 consecutive days. After recovery, a BDI score $<6$ was required for participation in the next step in the project. A consequence of the selection procedure was that all patients were studied for the first time when they were depressed. In contrast, 6 of 8 control subjects were first studied in summer. If appropriate, female patients were studied in the follicular phase of their menstrual cycle.

\section{Subjects}

From November 1997 until December 1999, 7 SAD patients ( 1 man and 6 women) and 8 control subjects (1 man and 7 women) were studied. Table 1 lists the respective scores (mean \pm SD) of patients and controls on the GHQ, SPAQ, and M-E questionnaire completed at the introduction meeting. For the winter studies, subjects entered the time isolation unit in the months October through March. The summer studies started in May and ended in August. One female control subject dropped out after finishing the protocol in summer and was replaced by another matched control for the winter experiment. Three patients and 3 controls smoked cigarettes.

Of the female patients, 1 had a regular natural menstrual cycle, 3 used combined oral contraceptives, 1 used a depot progesterone, and 1 was postmenopausal and used hormone replacement therapy during the winter. Consequently, 4 female patients could consistently be studied in the (pseudo-) follicular phase and 1 in the pseudoluteal phase of the menstrual cycle. The postmenopausal patient was studied in the pseudoluteal phase while depressed and in the pseudofollicular phase while remitted. Of the female controls, 2 had a regular natural menstrual cycle, 3 used combined oral contraceptives, and 2 were postmenopausal (one used estrogen replacement therapy). Except for the winter condition of the control subject who was matched with the postmenopausal patient, the times of participation of female controls were carefully matched for menstrual cycle phase. The mean baseline scores on the BDI, SIGH-SAD-SR, HDRS, and ATYP are summarized for each condition in Table 1. During their stay in the time isolation unit, 4 patients used NSAIDs (paracetamol): 2 while depressed ( 1 and $4 \times 500 \mathrm{mg}$, respectively), 1 while remitted $(3 \times 500 \mathrm{mg})$, and 1 in summer $(1 \times 500$ $\mathrm{mg})$.

\section{Design of the Protocol}

In each condition, patients and controls were subjected to a 10-day protocol. During the first 4 days, subjects adhered to a regular sleep-wake schedule at home. Adherence was verified by actimetry. The actimeter was worn continuously on the nondominant arm and was connected to a portable recorder (Bakker and Beersma, 1991). Sleep was scheduled from midnight till 0800. Subjects limited their coffee consumption to four cups a day and abstained from alcoholic beverages, heavy physical exercise, and daytime naps.

On day 4 at 1800, subjects entered the time isolation unit located in the Department of Psychiatry at Groningen Academic Hospital. The unit is shielded from all possible information on time of day. It comprises a small living room with a bed and a bathroom. After a period of familiarizing with the standard pro- 
Table 1. Characteristics (mean $\pm \mathrm{SD}$ ) of seasonal affective disorder patients and controls

\begin{tabular}{|c|c|c|c|c|c|}
\hline & \multicolumn{3}{|c|}{ Patients } & \multicolumn{2}{|c|}{ Controls } \\
\hline $\operatorname{Age}^{\mathrm{a}}$ & \multicolumn{3}{|c|}{$36.3 \pm 13.9$} & \multicolumn{2}{|c|}{$38.1 \pm 12.8$} \\
\hline GHQ & \multicolumn{3}{|c|}{$1.4 \pm 2.3$} & \multicolumn{2}{|c|}{$0.3 \pm 0.5$} \\
\hline $\operatorname{SPAQ}^{b}$ & \multicolumn{3}{|c|}{$16.7 \pm 3.6$} & \multicolumn{2}{|c|}{$3.5 \pm 1.8$} \\
\hline$M-E^{c}$ & & $44.7 \pm 10.4$ & & & \\
\hline Mood Scores at Baseline & $\begin{array}{c}\text { Depressed } \\
(\mathrm{n}=7)\end{array}$ & $\begin{array}{c}\text { Remitted } \\
(\mathrm{n}=7)\end{array}$ & $\begin{array}{c}\text { Summer } \\
(\mathrm{n}=7)\end{array}$ & $\begin{array}{l}\text { Winter } \\
(\mathrm{n}=7)\end{array}$ & $\begin{array}{c}\text { Summer } \\
(\mathrm{n}=7)\end{array}$ \\
\hline $\mathrm{BDI}^{\mathrm{d}}$ & $20.3 \pm 6.1$ & $1.7 \pm 1.8$ & $0.6 \pm 0.8$ & $0.3 \pm 0.8$ & $0.3 \pm 0.5$ \\
\hline SIGH-SAD-SR ${ }^{\mathrm{d}}$ & $32.3 \pm 11.8$ & $3.7 \pm 4.3$ & $1.1 \pm 1.9$ & $1.4 \pm 2.1$ & $1.6 \pm 1.6$ \\
\hline 21-item HDRS ${ }^{\mathrm{d}}$ & $21.3 \pm 7.9$ & $2.0 \pm 2.0$ & $0.9 \pm 1.5$ & $0.9 \pm 0.9$ & $1.3 \pm 1.5$ \\
\hline ATYP $^{d}$ & $11.0 \pm 5.4$ & $1.7 \pm 2.2$ & $0.3 \pm 0.5$ & $0.6 \pm 1.5$ & $0.3 \pm 0.8$ \\
\hline
\end{tabular}

NOTE: GHQ = General Health Questionnaire, SPAQ = Seasonal Personality Assessment Questionnaire, M-E = Morningness-Eveningness questionnaire, BDI = Beck Depression Inventory, SIGH-SAD-SR = Structured Interview Guide for the Hamilton Depression Rating ScaleSeasonal Affective Disorder-Self-Rating version, HDRS = Hamilton Depression Rating Scale, ATYP = Atypical Symptom Scale. a. Age at the start of the first experiment.

b. M-E scores < 30 and $>70$ correspond with evening and morning types, respectively (Horne and Ostberg, 1976).

c. Patients differed significantly from controls $(t$ test, $p=0.000)$.

d. An analysis of variance yielded $p<0.05$. Depressed patients differed significantly from all other groups; there were no significant differences between other groups.

cedures during wake time and a habituation night, the 120-h FD protocol started on day 5 at 0800. Subjects were blind to the experimental time schedule. The schedule consisted of six 20-h days. These artificial 20-h days were subdivided into epochs of $13.5 \mathrm{~h}$ of wakefulness in dim light ( $<10$ lux) and $6.5 \mathrm{~h}$ of darkness in which the subjects had to stay in bed. Each 20-h day had the same temporal structure. Staff members who were trained to communicate no information on time of day, announced the moments of rising, showering, meals, completing psychometric test batteries, and going to bed. Besides the scheduled activities, subjects could engage in all kinds of activities such as watching videos, listening to music, and so on. A maximum of four caffeine-containing drinks was allowed only during the subjective morning. On average, subjects consumed 2.6 caffeinated beverages per day of the protocol. There was no significant effect of time in study or between experimental conditions. Throughout their stay in the time isolation unit, subjects were continuously observed through an infrared television camera.

\section{Melatonin}

On days 4 and 9 of the protocol, from 1700 until midnight and from 1700 until 0200 , respectively, saliva was sampled hourly for the determination of melatonin concentration. In two patients, saliva was collected in small plastic containers. In all other subjects, it was sampled by means of Salivettes (Sarstedt, Nümbrecht, Germany) with a cotton swab. Subjects were asked to remain seated during the $15 \mathrm{~min}$ prior to the saliva collection. All samples were frozen at $\leq-18$ ${ }^{\circ} \mathrm{C}$ immediately afterward. After each saliva sample, eating (with the exception of chocolate and bananas), drinking, and smoking were allowed for $15 \mathrm{~min}$. Then, subjects thoroughly rinsed their mouth with water.

Melatonin concentrations were measured by means of radioimmunoassay (Bühlmann, Allschwil, Switzerland), which was performed at the laboratory of the hospital De Gelderse Vallei in Bennekom, the Netherlands. In this laboratory, the following assay parameters were obtained: a limit of detection of 0.5 $\mathrm{pg} / \mathrm{mL}$, an intra-assay variation of covariance of $8.78 \%$ (mean melatonin concentration $8.65 \mathrm{pg} / \mathrm{mL}, n=$ $26)$, and an interassay variation of covariance in samples with a low melatonin concentration of $9.6 \%$ (mean $1.98 \mathrm{pg} / \mathrm{mL}, n=30$ ) and in samples with a high concentration of $9.8 \%$ (mean $14.76 \mathrm{pg} / \mathrm{mL}, n=30)$. For each experiment, melatonin values were expressed as a percentage of the maximum value observed on day 4 . DLMO was defined as the time at which the normalized melatonin curve crossed the $25 \%$ level. Because the body temperature recordings confirmed that five circadian cycles had passed between the two DLMOs, an estimate of the circadian rhythm period $(\tau)$ could be obtained by dividing the epoch between the two DLMOs by five. 


\section{Core Body Temperature}

Throughout the entire stay in the time isolation unit, core body temperature was measured continuously with a calibrated rectal thermometer (Yellow Springs Instrument Company, Yellow Springs, $\mathrm{OH}$, USA). The data were stored at 1-min intervals by a portable recorder (Bakker and Beersma, 1991). Those parts of the temperature curve that were considered unreliable due to technical failures were not used for further analysis. Intervals of missing data shorter than $1.5 \mathrm{~h}$ in duration were linearly interpolated, and intervals longer than $1.5 \mathrm{~h}$ were regarded as missing data.

To identify the endogenous circadian temperature curve, the following mathematical procedure was used. It is based on the assumption that the raw temperature data obtained during the 120-h FD protocol represent the sum of the exogenous sleep-wakeinduced curve and the endogenous circadian curve. First, the raw data of the 6 consecutive 20-h subjective days were averaged to obtain an estimation of the mean sleep-wake-related course of body temperature. With this procedure, the circadian contribution to the raw temperature data is roughly averaged out, as each subjective $20-\mathrm{h}$ day has the same temporal structure and all scheduled activities occur at all circadian phases. To obtain an estimation of the endogenous circadian temperature curve, the obtained mean sleep-wake-related temperature curve was subtracted from the raw curve. Subsequently, the resulting curve was divided into epochs with the length of the melatonin-derived $\tau$ value and then averaged (starting from 0800 on day 4 of the protocol). Due to the fact that the melatonin-derived $\tau$ values differ from $24 \mathrm{~h}$, the pacemaker-related contribution was not averaged out completely during the first step of the mathematical procedure. To establish a better estimation of the sleep-wake-related contribution to the temperature curve, the obtained average circadian temperature curve was subtracted from the original raw data. The resulting curve was again divided into 20-h epochs and averaged, and so on. This procedure was repeated until a stable estimation of the circadian temperature curve was obtained (see Hiddinga et al., 1997). On the basis of that endogenous component, the timing of the minimum on the first day in the FD protocol was determined as a measure of baseline phase. For that purpose, the endogenous component was extrapolated backward to the first day of the protocol. The clock time was determined of the midpoint between the upward and downward crossings through the average value of the temperature by means of a 1-h moving average procedure. A measure of amplitude was obtained for the sleep-wakeinduced variation and for the pacemaker-induced variation of body temperature. For the sleep-wakeinduced variation, this was done by computing the standard deviation of the data of the last $6.5 \mathrm{~h}$ of wakefulness and the $6.5 \mathrm{~h}$ allowed for sleep. In this way, the influence of showering on the amplitude is excluded. As a measure of amplitude of the circadian pacemaker-related variation, the standard deviation of all data within one cycle of the pacemaker-induced temperature variation curve is calculated.

\section{Polysomnography}

From each subjective night, a polysomnographical (PSG) recording was obtained. Location of electrodes and scoring criteria were according to the standards defined by Rechtschaffen and Kales (1968). The PSG recordings were scored in 30-sec intervals with the aid of VitaPort software (TEMEC Instruments, Kerkrade, the Netherlands) by three raters. The average agreement between raters was $95.4 \%$ (range: $94.1 \%$ to $96.3 \%$ ), with an average largest disagreement interval of $3 \mathrm{~min}$ (range: 1.5 to $4 \mathrm{~min}$ ). Total sleep time (TST) per sleeping period was calculated by adding the total amount of minutes in sleep stages 1,2,3,4, and REM.

\section{Statistical Procedures}

Power analyses were performed to estimate the smallest detectable differences by calculating $\delta$ from the formula $n \geq 2(\sigma / \delta)^{2}\left\{t_{\alpha[v]}+t_{2(1-P)[v]}\right\}^{2}$, with a power $P$ $=0.80$. In this formula, $n$ represents the number of replications, $\sigma$ the true standard deviation, $\delta$ the smallest desired difference to detect, $\alpha$ the level of significance, $P$ the intended power of the test, $v$ the degrees of freedom $(v=a(n-1))$, and $a$ the number of populations; $t_{0.05[12]}=2.179$ and $t_{0.4[12]}=0.873$ (Sokal and Rohlf, 1981).

The data derived from melatonin and temperature recordings were analyzed statistically by means of paired-samples tests. Analysis of variance (ANOVA) with repeated measures was applied to evaluate TST. The Spearman correlation coefficient was computed to evaluate the association between the average level of core body temperature and the amplitude of the observed circadian temperature component. The 
Table 2. Circadian and sleep-wake-related characteristics derived from core body temperature and melatonin

\begin{tabular}{|c|c|c|c|c|c|}
\hline & \multirow{2}{*}{$\underset{\tau^{\mathrm{a}}}{\text { Melatonin }}$} & \multicolumn{4}{|c|}{ Core Body Temperature } \\
\hline & & Mean $^{\mathrm{b}}$ & Sleep-Wake Amplitude ${ }^{\mathrm{c}}$ & Circadian Amplitude $\mathrm{d}^{\mathrm{d}}$ & Circadian Phase $e^{\mathrm{e}}$ \\
\hline Patients (depressed; $n=7$ ) & $24.03 \pm 0.07$ & $37.23 \pm 0.10$ & $0.17 \pm 0.020$ & $0.14 \pm 0.0097$ & $4: 31 \pm 0: 23$ \\
\hline Patients (remitted; $n=7$ ) & $24.13 \pm 0.08$ & $37.22 \pm 0.07$ & $0.16 \pm 0.021$ & $0.12 \pm 0.0060$ & $4: 26 \pm 0: 29$ \\
\hline Patients (summer; $n=7$ ) & $24.17 \pm 0.1$ & $37.16 \pm 0.11$ & $0.15 \pm 0.019$ & $0.16 \pm 0.0174$ & $4: 43 \pm 0: 27$ \\
\hline Controls (winter; $n=7$ ) & $24.22 \pm 0.08$ & $37.00 \pm 0.11$ & $0.19 \pm 0.024$ & $0.17 \pm 0.0150$ & $5: 12 \pm 0: 42$ \\
\hline Controls (summer; $n=7$ ) & $24.27 \pm 0.1$ & $36.95 \pm 0.15$ & $0.17 \pm 0.021$ & $0.18 \pm 0.0211$ & $4: 47 \pm 0: 22$ \\
\hline \multicolumn{6}{|l|}{ Paired-samples $t$ test $^{\mathrm{f}}$} \\
\hline Patient (depressed vs. remitted) & 0.282 & 0.984 & 0.431 & 0.240 & 0.621 \\
\hline Patient (depressed vs. summer) & 0.259 & 0.130 & 0.075 & 0.160 & 0.669 \\
\hline Patient (remitted vs. summer) & 0.720 & 0.322 & 0.243 & 0.093 & 0.564 \\
\hline Patient (depressed) vs. control (winter) & 0.129 & 0.163 & 0.473 & 0.050 & 0.481 \\
\hline Patient (remitted) vs. control (winter) & 0.327 & 0.156 & 0.157 & 0.031 & 0.462 \\
\hline Patient (summer) vs. control (summer) & 0.533 & 0.218 & 0.521 & 0.464 & 0.929 \\
\hline Control (winter) vs. control (summer) & 0.563 & 0.726 & 0.408 & 0.634 & 0.464 \\
\hline
\end{tabular}

a. The circadian rhythm period $(\tau)$ in hours (mean \pm SEM) derived from the timing of the dim-light melatonin onset obtained on the first (day 4 ) and the last (day 9) evening in time isolation.

b. Mean $\left( \pm\right.$ SEM) level of core body temperature $\left({ }^{\circ} \mathrm{C}\right)$ calculated by averaging the temperature data of the last $6.5 \mathrm{~h}$ of wakefulness and the 6.5 - $\mathrm{h}$ sleeping period.

c. Amplitude (mean \pm SEM) of the sleep-wake-related variation of body temperature $\left({ }^{\circ} \mathrm{C}\right)$ defined as the standard deviation calculated over the last $6.5 \mathrm{~h}$ of wakefulness and the 6.5-h sleeping period.

d. Amplitude (mean \pm SEM) of the circadian variation of body temperature $\left({ }^{\circ} \mathrm{C}\right.$ ) defined as the standard deviation calculated over the total circadian variation of body temperature.

e. Timing of the circadian temperature minimum (mean \pm SEM) on the first morning in time isolation, determined by extrapolating backward from the educed circadian pacemaker-related curves to the first day of the protocol.

f. $p$ values obtained by two-tailed paired-samples $t$ tests between the various conditions in which patients and controls participated.

analyses were two-tailed, and statistical significance was established at $p<0.05$. With this procedure, no correction is applied to account for multiple testing.

\section{RESULTS}

\section{The Circadian Period, $\tau$, Based on Melatonin Data}

In each condition, melatonin concentrations showed a gradual increase. As mentioned earlier, DLMO was defined as the time at which melatonin production reached $25 \%$ of the maximal production on day 4 . In 2 of the remitted patients, the initial melatonin production in the second sampling period (day 9) exceeded this $25 \%$ criterion. Therefore, in these subjects the criterion was raised to $50 \%$. The exact choice of the criterion level has little influence on the resulting determination of the period of the signal, if only the level occurs at a steep area of the curves. However, because the choice of the criterion value does influence the assessment of phase, DLMO times were exclusively used for the estimation of the circadian period $(\tau)$. All $\tau$ values approximated $24 \mathrm{~h}$. Between conditions, no significant differences in $\tau$ values were found (Table 2).

\section{Evaluation of the Sleep-Wake-Related and Pacemaker-Related Temperature Components}

Of the core body temperature data, $9.61 \%$ were missing due to technical problems and matters of hygiene. The analysis described earlier provided the contributions of the circadian pacemaker and those of the sleep-wake cycle to the raw temperature curves. Figure 1 displays these contributions per condition.

The sleep-wake-induced temperature curve shows a remarkable resemblance in the various conditions. After waking up, body temperature shows a peak induced by showering. Subsequently, it drops and stays relatively stable during the remaining hours of wakefulness. At the start of the 6.5-h period in bed, a sharp decrease in temperature can be observed. The mean level of body temperature (listed in Table 2) was calculated by averaging the data of the last $6.5 \mathrm{~h}$ of wakefulness and the $6.5 \mathrm{~h}$ of sleep. In this way, the highly variable influence of showering on the mean was excluded. Although not significantly, mean body temperature of the patients was slightly higher during the winter than during the summer and also slightly higher than that of controls. The amplitudes of the sleep-wake-induced variation of body temperature (see Table 2) showed no differences between patients and controls. 


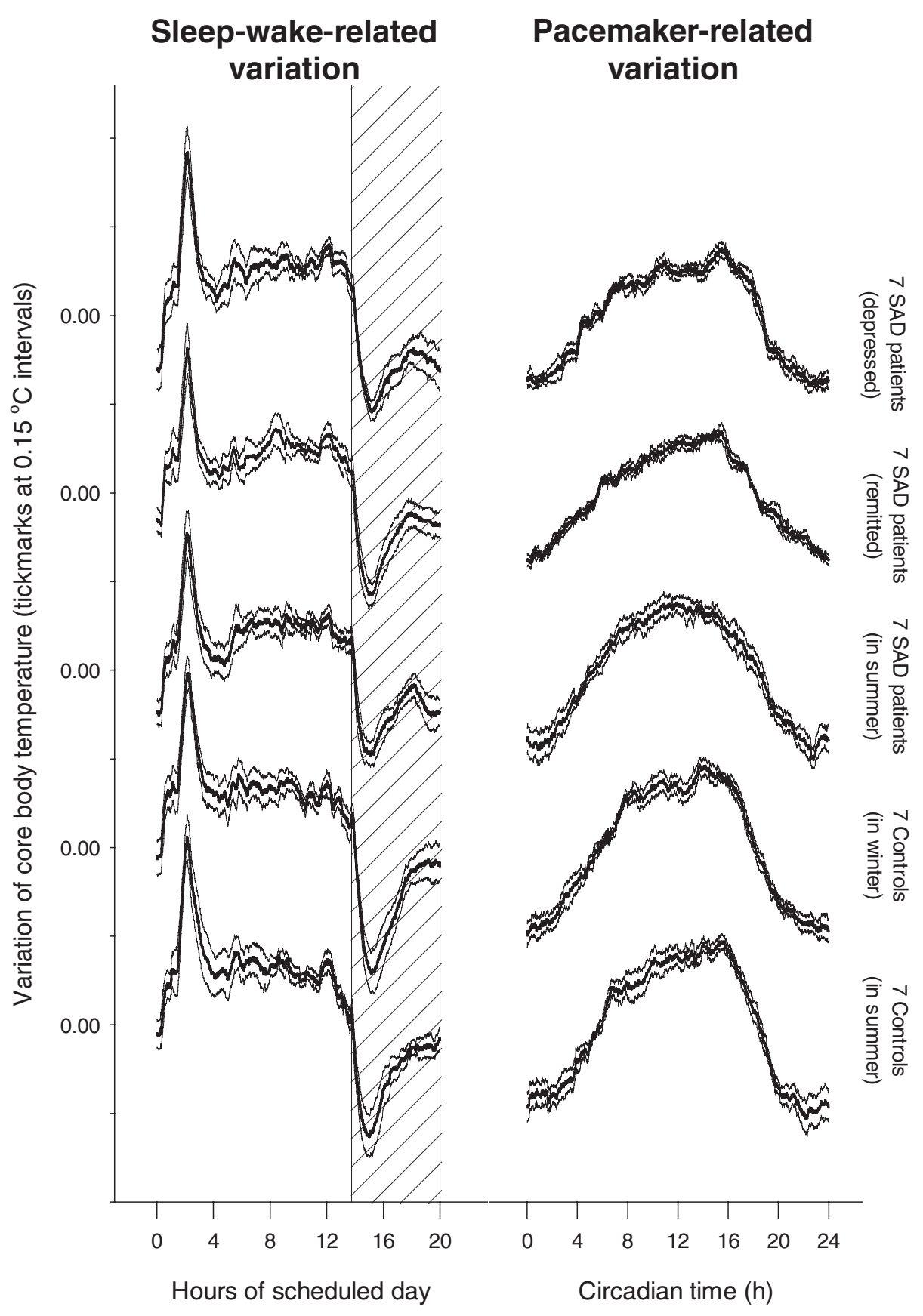

Figure 1. Sleep-wake-related and pacemaker-related variation of core body temperature obtained in a 120-h forced desynchrony experiment. In each condition, 7 seasonal affective disorder (SAD) patients and 7 matched controls participated. The curves, plotted as deviation from the mean, depict the mean ( \pm SEM) temperature variation in each condition. The shaded area indicates the 6.5-h sleeping period within the 20-h subjective day. Circadian time is defined here as the time from one minimum in the circadian component of core body temperature to the next. This interval is divided into 24 equal circadian-hour intervals.

Figure 1 also shows the circadian variation of body temperature plotted relative to the moment at which the endogenous circadian temperature reaches its minimum. A measure of the amplitude of the pacemakerinduced variation of body temperature is presented in
Table 2. Only comparisons of the depressed patients and the remitted patients with the controls in winter showed statistically significant differences $(p=0.050$ and $p=0.031$ ). The patients' amplitude turned out to be smaller. In each condition, the core body tempera- 


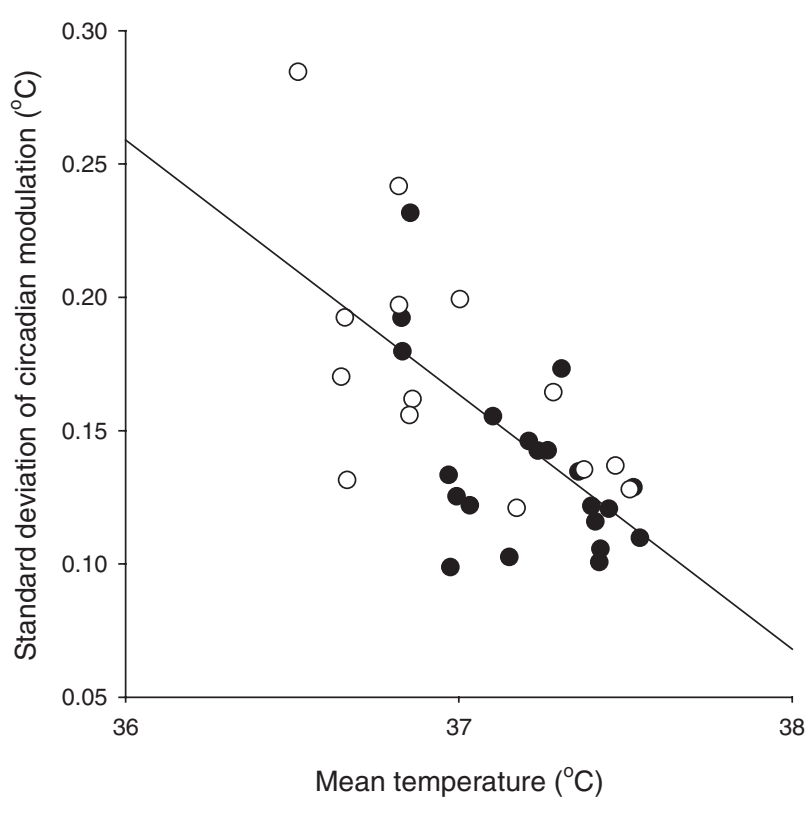

Figure 2. Correlation between average level of core body temperature and circadian amplitude. Shown is the standard deviation (SD) of the circadian modulation (see Fig. 1, right panel) as a function of the average level of body temperature. The SD of the circadian modulation is proportional to the circadian amplitude (for a sine function, the SD equals $1 / 2 \sqrt{2}$ times the circadian amplitude). Filled circles depict the data obtained in patients $(n=21)$, and open circles depict data obtained in controls $(n=14)$. The diagonal line represents the regression line. Correlation coefficient (Spearman rho) of all conditions combined is $-0.627(p=0.000)$, of the patient group is $-0.508(p=0.019)$, and of the control group is $0.604(p=0.022)$.

ture curve reached its minimum in the early morning (Table 2). Between conditions, no significant differences in the timing of this minimum were observed.

In Figure 2, a measure of the amplitude of the pacemaker-induced temperature curve is plotted against the average level of body temperature for all subjects in all conditions. The data reveal a significant correlation between the average level of body temperature and the amplitude of the pacemaker-related temperature component (Spearman rho $=-0.627, p=$ 0.000). A significant correlation between these variables was also found in the patients and controls separately (Spearman rho $=-0.508$ and -0.604 , respectively, $p=0.019$ and $p=0.022$ ). This suggests that the higher the average level of body temperature, the smaller is the amplitude of the endogenous circadian temperature component.

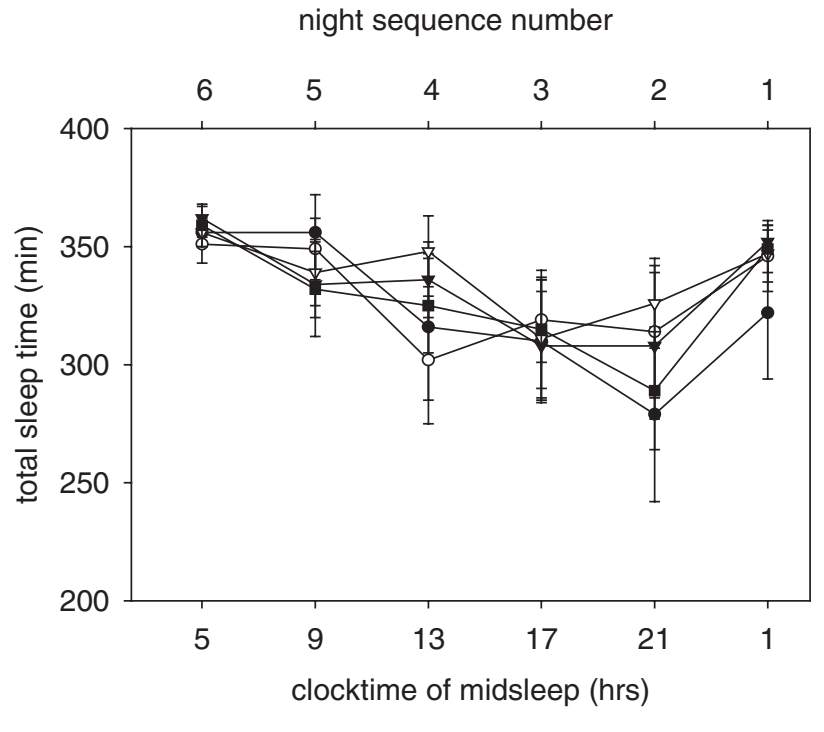

Figure 3. Duration of total sleep time as a function of the clock time at midsleep. Filled circles represent patients while depressed, open circles represent patients while remitted, and filled triangles represent patients in summer. Open triangles represent controls in winter, and filled squares represent controls in summer.

\section{Polysomnography}

The mean TSTs in the subjective nights in FD are summarized in Figure 3. In the data of all conditions combined, ANOVA with repeated measures yielded a significant effect of night number in FD on TST, $F(5$, $26)=6.872, p=0.000$. However, no significant interaction between night number and condition was detected, $F(20,116)=0.667, p=0.851$. Separate ANOVAs to compare the various conditions in which patients and controls were studied did not reveal significant differences either. Because night sequence number is strictly linked to the circadian phase of the respective sleep episode, the data show that TST varies systematically as a function of circadian phase.

\section{DISCUSSION}

The aim of the present FD study was to compare the characteristics of the endogenous circadian pacemaker of SAD patients with those of matched controls. It is concluded that the circadian pacemakers of patients and controls have a similar period, in both winter and summer and irrespective of the 
symptomatological state of the patients. The same applies to the phase of the endogenous circadian cycle after 4 days of restricting sleep to the interval between midnight and 0800. The only possible difference detected in our study concerns the amplitude of the endogenous circadian temperature component. Compared to that found in controls, the circadian amplitude appeared to be slightly smaller in SAD patients in winter, both during a depressive episode and in the recovered state.

Previously, we have reported on the circadian and sleep-wake cycle-related variations of core body temperature observed in one 54-year-old male SAD patient studied in a 120-h FD protocol both while depressed and while remitted after light therapy (Koorengevel et al., 2000). This subject, who is included in the present analysis, provided evidence for a phase delay of the circadian pacemaker during a depressive episode. This is in contrast with the enlarged data set in which no evidence for pacemaker disturbances in SAD was found. In addition, in the present study DLMO values were used instead of body temperature curves to estimate circadian period. As expected on the basis of reduced sensitivity to masking, the melatonin-derived $\tau$ values showed much smaller standard deviations in comparison to the temperature-derived values. This is the reason that melatonin-derived $\tau$ values were used. In contrast, however, circadian phase was derived on the basis of the body temperature curves. This was done because melatonin-derived phase angles depend strongly on the criterion melatonin concentration value used to define DLMO. Data on the timing of the offset of melatonin production are required in addition to onset data in order to determine the phase position of the melatonin profile. In the absence of those offset data, body temperature curves were used to determine circadian phase.

\section{Endogenous Circadian Period}

DLMOs obtained in the first and last evening in time isolation showed an endogenous circadian period $(\tau)$ of approximately $24 \mathrm{~h}$ in each condition. Just like body temperature, salivary and plasma melatonin concentrations exhibit a distinct circadian rhythm. Yet, apart from an endogenous circadian modulation, the secretion of melatonin has also been found to depend on the timing of the sleep-wake cycle (or the dim light:dark cycle) (Gordijn et al., 1999; Ritz-
De Cecco et al., 1999; Wyatt et al., 1999). In the present study, a small difference occurred in elapsed hours of wakefulness on the 2 days of saliva collection. This may have induced a small overestimation of $\tau$ in all conditions. However, the $\tau$ values observed in SAD patients and controls are similar to those computed with temperature or melatonin data in other FD studies on healthy subjects in 20-h-day protocols $(24.30 \mathrm{~h}$, Hiddinga et al., 1997; 24.1 to $24.2 \mathrm{~h}$, Wyatt et al., 1999) as well as in 28 -h-day protocols $(24.18 \mathrm{~h}$, Czeisler et al., 1999; 24.3 to 24.35 h, Carskadon et al., 1999). Such a similarity between our results and those of longerlasting FD protocols further suggests that the duration of the experiment has little influence on the results. Socalled aftereffects, by which a pacemaker would need time to switch from its entrained 24-h state to its intrinsic free run, apparently are not very strong in humans.

The phase position of a circadian process during entrainment depends on the intrinsic period length, a long period leading to a late phase position. In the absence of a difference in $\tau$ between SAD patients and controls, the suggested phase delay of SAD (Lewy, Sack, Singer, et al., 1987) cannot be attributed to a longer intrinsic period.

\section{Circadian Phase}

Unlike data from some treatment (Lewy, Sack, Miller, et al., 1987; Sack et al., 1990; Lewy et al., 1998) and constant routine (Dahl et al., 1993; Avery et al., 1997) studies, our data did not show abnormalities of circadian phase. No differences were found between the various conditions with respect to the timing of the circadian temperature minimum. Although this may be due to the synchronizing effects of the instructions concerning the timing of sleep on the days prior to FD, it is also possible that the small sample size of the present study is responsible. This will be discussed below. Nevertheless, the data do not confirm a phase delay in depressed SAD patients (Lewy, Sack, Singer, et al., 1987). The implications of the present study for the phase-shift hypothesis (i.e., whether a shift in phase of the circadian pacemaker relative to the sleep-wake cycle influences mood) will be discussed elsewhere (Koorengevel et al., unpublished data).

\section{Circadian Amplitude}

In winter, a smaller endogenous circadian amplitude was observed in depressed and remitted patients 
compared to controls. Average level of body temperature and circadian amplitude were negatively correlated in the total data set as well as in the separate data sets on patients and controls. Consequently, the relatively small circadian amplitude in SAD patients might be explained by the relatively high average body temperature. Because body temperature in homeothermic organisms is regulated within a restricted range of values, it is conceivable that because of ceiling effects, a higher body temperature level leads to a smaller variation. Therefore, a decrease of the endogenous circadian amplitude of body temperature in depressed and remitted patients could be the consequence of a higher level of body temperature instead of the result of a disturbance of the circadian system.

\section{Influence of Menstrual Cycle and NSAIDs on Body Temperature}

To control for the influences of gender and for the influences of gonadal hormones and oral contraceptives on body temperature specifically (Wright and Badia, 1999), in each experiment female subjects were studied in the same phase of the menstrual cycle (which was the follicular phase if appropriate). Apart from the sporadic use of NSAIDs by female patients in all three conditions, all subjects were free of medication. Studies of the effects of NSAIDs on the body temperature have been inconclusive. NSAIDs have been reported to yield no effects on the circadian variation of body temperature (Scales et al., 1988). However, another study has observed an attenuation of the decrease in tympanic temperature and a suppression of melatonin secretion for some hours after nighttime administration of an NSAID (Murphy et al., 1996). Hence, small effects of the incidentally used NSAIDs cannot be fully excluded.

\section{Power of the Study}

A disadvantage of the present study is the rather small sample size. This is due to the high demands on the subjects living in isolation from their normal environment for such a long time. Small sample sizes have consequences for the smallest detectable difference $(\delta)$ (Sokal and Rohlf, 1981) of each variable under study. A post hoc power analysis of the obtained results, under the assumption of a power value of 0.8 , revealed a smallest detectable difference $(\delta)$ of $(1) 0.45^{\circ} \mathrm{C}$ in the average core body temperature level, (2) $22 \mathrm{~min}$ in the melatonin-derived $\tau$ values, (3) $0.059^{\circ} \mathrm{C}$ in the amplitude of the endogenous circadian temperature component, and (4) $123 \mathrm{~min}$ in the timing of the circadian temperature minimum. Thus, the absence of differences in phase position might be attributed to a lack of power, whereas the obtained values for $\tau$ and amplitude might be accurate enough to enable adequate comparisons.

Another weakness of the present study is the relatively large number of statistical tests performed, which increases the chance of finding significant results. Only the data on circadian amplitude yielded statistically significant differences between SAD patients (both when depressed and remitted) and controls in winter. Although SAD patients might generally exhibit a smaller circadian amplitude in winter, it is also possible that this result is due to chance variations in our sample. However, we have decided to perform multiple tests in order to avoid missing possible differences in pacemaker characteristics between patients and controls.

\section{Clinical State}

In all experiments, both patients and controls tolerated the protocol quite well. Comparison of the average scores on the BDI and SIGH-SAD-SR completed prior to and directly following the 10-day protocol revealed a small deterioration of mood in both patients and controls. Thus, the FD protocol did not induce a strong effect on mood in patients. In one FD study, an interaction between pacemaker and sleepwake cycle-related variation was demonstrated in the regulation of mood in healthy subjects (Boivin et al., 1997). The present study revealed a similar modulation of self-rated mood in the course of the experiment in all conditions (Koorengevel et al., unpublished data).

PSG data did show an effect of night sequence number on TST. This effect did not differ between conditions. Like the TST data from the present study, PSG data obtained in another FD study using 20-h subjective days showed highest and lowest sleep efficiency when sleep was centered on the minimum of core body temperature and $16 \mathrm{~h}$ later, respectively (Wyatt et al., 1999).

Thus, the 120-h FD protocol not only induced similar effects on sleep in patients and controls-it also 
hardly affected the overall clinical state. It can therefore be concluded that FD provides an adequate tool in the study of the circadian system in depressed patients.

In summary, this 120-h FD study on SAD suggests that period and phase of the circadian pacemaker assessed by measuring core body temperature and melatonin do not differ in (1) depressed and remitted SAD patients versus controls in winter, (2) in SAD patients versus controls in summer, and (3) in SAD patients and controls in winter versus summer. In addition, the significant correlation between average level of temperature and endogenous circadian amplitude suggests that in SAD patients, thermoregulation might be disturbed in the winter season.

\section{ACKNOWLEDGMENTS}

This research was supported by the Nederlandse Gasunie B.V., the Medical Faculty of the University of Groningen, the Academic Hospital Groningen, the Ministry of Health, and the National Fund of Mental Health. The authors are grateful to the subjects for their participation; to Iteke te Riet, Ybe Meesters, Gerda Bloem, Marijke Gordijn, Theodoor van Maaren, Maarten Langenberg, Jan van Dijken, Marieke Kienhuis, Wendy Blom, Louise Dols, and Jacqueline Reisel for their contribution to the performance of the study; to Camiel Elsinga for his help in scoring the polysomnographical recordings; and to J.J. Veeken and coworkers of the laboratory of the Hospital De Gelderse Vallei for the melatonin analysis.

\section{REFERENCES}

American Psychiatric Association (1994) Diagnostic and Statistical Manual of Mental Disorders, 4th ed, Washington, DC, American Psychiatric Press.

Aschoff J (1965) Circadian rhythms in man: A self-sustained oscillator with a inherent frequency underlies human 24hour periodicity. Science 148:1427-1432.

Avery DH, Dahl K, Savage MV, Brengelmann GL, Larsen LH, Kenny MA, et al (1997) Circadian temperature and cortisol rhythms during a constant routine are phasedelayed in hypersomnic winter depression. Biol Psychiatry 41:1109-1123.

Bakker JO and Beersma DGM (1991) A digital 3-channel recorder for long term registrations. Sleep Res 20A:485.

Beck AT, Rush AJ, Shaw BF, and Emergy G (1979) Cognitive Therapy of Depression, New York, NY, John Wiley \& Sons.

Boivin DB, Czeisler CA, Dijk DJ, Duffy JF, Folkard S, Minors DS, et al (1997) Complex interaction of the sleep-wake cycle and circadian phase modulates mood in healthy subjects. Arch Gen Psychiatry 54:145-152.

Boivin DB, Duffy JF, Kronauer RE, and Czeisler CA (1996) Dose-response relationships for resetting of human circadian clock by light. Nature 379:540-542.

Brendel DH, Reynolds CF, Jennings JR, Hoch CC, Monk TH, Berman SR, et al (1990) Sleep stage physiology, mood, and vigilance responses to total sleep deprivation in healthy 80-year-olds and 20-year-olds. Psychophysiology 27:677-685.

Carskadon MA, Labyak SE, Acebo C, and Seifer R (1999) Intrinsic circadian period of adolescent humans measured in conditions of forced desynchrony. Neurosci Lett 260:129-132.

Checkley SA, Murphy DG, Abbas M, Marks M, Winton F, Palazidou E, et al (1993) Melatonin rhythms in seasonal affective disorder. Br J Psychiatry 163:332-337.

Czeisler CA, Allan JS, and Kronauer RE (1986) A method to assess the intrinsic period of the endogenous circadian oscillator. Sleep Res 15:266.

Czeisler CA, Brown EN, Ronda JM, Kronauer RE, Richardson GS, and Freitag WO (1985) A clinical method to assess the endogenous circadian phase (ECP) of the deep circadian oscillator in man. Sleep Res 14:295.

Czeisler CA, Duffy JF, Shanahan TL, Brown EN, Mitchell JF, Rimmer DW, et al (1999) Stability, precision, and near-24hour period of the human circadian pacemaker. Science 284:2177-2181.

Czeisler CA, Kronauer RE, Mooney JJ, Anderson JL, and Allan JS (1987) Biologic rhythm disorders, depression, and phototherapy: A new hypothesis. Psychiatric Clin North Am 10:687-709.

Dahl K, Avery DH, Lewy AJ, Savage MV, Brengelmann GL, Larsen LH, et al (1993) Dim light melatonin onset and circadian temperature during a constant routine in hypersomnic winter depression. Acta Psychiatr Scand 88:60-66.

Dijk DJ, Duffy JN, and Czeisler CA (1992) Circadian and sleep/wake dependent aspects of subjective alertness and cognitive performance. J Sleep Res 1:112-117.

Eastman CI, Gallo LC, Lahmeyer HW, and Fogg LF (1993) The circadian rhythm of temperature during light treatment for winter depression. Biol Psychiatry 34:210-220.

Goldberg D and Williams PA (1988) User's Guide to the General Health Questionnaire, Windsor, UK, NDER-Nelson.

Gordijn MC, Beersma DG, Korte HJ, and van den Hoofdakker RH (1999) Effects of light exposure and sleep displacement on dim light melatonin onset. J Sleep Res 8:163-174.

Graw P, Haug HJ, Leonardt G, and Wirz-Justice A (1998) Sleep deprivation response in seasonal affective disorder during a 40-h constant routine. J Affective Disord 48:6974.

Hiddinga AE, Beersma DG, and van den Hoofdakker RH (1997) Endogenous and exogenous components in the circadian variation of core body temperature in humans. J Sleep Res 6:156-163.

Honma K and Honma S (1988) A human phase response curve for bright light pulses. Jpn J Psychiatry Neurol 42:167-168. 
Horne JA and Ostberg O (1976) A self-assessment questionnaire to determine morningness-eveningness in human circadian rhythms. Int J Chronobiol 4:97-110.

Jewett ME, Rimmer DW, Duffy JF, Klerman EB, Kronauer RE, and Czeisler CA (1997) Human circadian pacemaker is sensitive to light throughout subjective day without evidence of transients. Am J Physiol 273:R1800-R1809.

Kasper S, Wehr TA, Bartko JJ, Gaist PA, and Rosenthal NE (1989) Epidemiological findings of seasonal changes in mood and behavior: A telephone survey of Montgomery County, Maryland. Arch Gen Psychiatry 46:823-833.

Kleitman N and Kleitman E (1953) Effect of non-twentyfour-hour routines of living on oral temperature and heart rate. J Appl Physiol 6:283-291.

Koorengevel KM, Beersma DGM, Gordijn MCM, den Boer JA, and van den Hoofdakker RH (2000) Body temperature and mood variations during forced desynchronization in winter depression: A preliminary report. Biol Psychiatry 47:355-358.

Levendosky AA, Joseph-Vanderpool JR, Hardin T, Sorek E, and Rosenthal NE (1991) Core body temperature in patients with seasonal affective disorder and normal controls in summer and winter. Biol Psychiatry 29:524-534.

Lewy AJ, Bauer VK, Cutler NL, Sack RL, Ahmed S, Thomas $\mathrm{KH}$, et al (1998) Morning vs evening light treatment of patients with winter depression. Arch Gen Psychiatry 55:890-896.

Lewy AJ, Sack RL, Miller LS, and Hoban TM (1987) Antidepressant and circadian phase-shifting effects of light. Science 235:352-354.

Lewy AJ, Sack RL, and Singer CM (1985) Immediate and delayed effects of bright light on human melatonin production: Shifting "dawn" and "dusk" shifts the dim light melatonin onset (DLMO). Ann NY Acad Sci 453:253-259.

Lewy AJ, Sack RL, Singer CM, and White DM (1987) The phase shift hypothesis for bright light's therapeutic mechanism of action: Theoretical considerations and experimental evidence. Psychopharmacol Bull 23:349353.

Lewy AJ, Wehr TA, Goodwin FK, Newsome DA, and Markey SP (1980) Light suppresses melatonin secretion in humans. Science 210:1267-1269.

Mills JN, Minors DS, and Waterhouse JM (1978) Adaptation to abrupt time shifts of the oscillator(s) controlling human circadian rhythms. J Physiol 285:455-470.

Minors DS, Waterhouse JM, and Wirz-Justice A (1991) A human phase-response curve to light. Neurosci Lett 133:36-40.

Murphy PJ, Myers BL, and Badia P (1996) Nonsteroidal antiinflammatory drugs alter body temperature and suppress melatonin in humans. Physiol Behav 59:133-139.

Rechtschaffen A and Kales A (1968) A Manual of Standardized Terminology, Techniques, and Scoring System for Sleep Stages of Human Subjects, Washington, DC, Government Printing Office.

Ritz-De Cecco A, Jewett ME, Wyatt JK, Kronauer RE, Czeisler CA, and Dijk DJ (1999) Plasma melatonin rhythm in humans during a 20-h forced desynchrony protocol. Sleep Res Online 2(suppl 1):620.
Rosenthal NE, Genhart M, Sack DA, Skwerer RG, and Wehr TA (1987) Seasonal affective disorder and its relevance for the understanding and treatment of bulimia. In The Psychology of Bulimia, JJ Hudson and HG Pope Jr, eds, pp 205228, Washington, DC, American Psychiatric Press.

Rosenthal NE, Levendosky AA, Skwerer RG, JosephVanderpool JR, Kelly KA, Hardin T, et al (1990) Effects of light treatment on core body temperature in seasonal affective disorder. Biol Psychiatry 27:39-50.

Rosenthal NE, Sack DA, Gillin JC, Lewy AJ, Goodwin FK, Davenport Y, Mueller PS, et al (1984) Seasonal affective disorder: A description of the syndrome and preliminary findings with light therapy. Arch Gen Psychiatry 41:7280.

Sack RL, Lewy AJ, White DM, Singer CM, Fireman MJ, and Vandiver R (1990) Morning vs evening light treatment for winter depression: Evidence that the therapeutic effects of light are mediated by circadian phase shifts. Arch Gen Psychiatry 47:343-351.

Scales WE, Vander AJ, Brown MB, and Kluger MJ (1988) Human circadian rhythms in temperature, trace metals, and blood variables. J Appl Physiol 65:1840-1846.

Schwartz PJ, Rosenthal NE, Turner EH, Drake CL, Liberty V, and Wehr TA (1997) Seasonal variation in core temperature regulation during sleep in patients with winter seasonal affective disorder. Biol Psychiatry 42:122-131.

Sokal RR and Rohlf IJ (1981) Biometry: The Principles and Practice of Statistics in Biological Research, 2nd ed, New York, NY, WH Freeman and Company.

Terman JS, Terman M, Lo ES, and Cooper TB (2001) Circadian time of morning light administration and therapeutic response in winter depression. Arch Gen Psychiatry 58:69-75.

Thompson C, Childs PA, Martin NJ, Rodin I, and Smythe PJ (1997) Effects of morning phototherapy on circadian markers in seasonal affective disorder. Br J Psychiatry 170:431-435.

Van Esseveldt KE, Lehman MN, and Boer GJ (2000) The suprachiasmatic nucleus and the circadian time-keeping system revisited. Brain Res Rev 33:34-77.

Williams JBW, Link MJ, Rosenthal NE, Amira L, and Terman M (1992) Structured Interview Guide for the Hamilton Rating Scale-Seasonal Affective Disorder Version (SIGH-SAD, SIGH-SAD-SR, Self-Rating Version), New York, NY, New York State Psychiatric Institute.

Wirz-Justice A, Kräuchi K, Brunner DP, Graw P, Haug HJ, Leonardt G, et al (1995) Circadian rhythms and sleep regulation in seasonal affective disorder. Acta Neuropsychiatrica 7:41-43.

Wright KP and Badia P (1999) Effects of menstrual cycle phase and oral contraceptives on alertness, cognitive performance, and circadian rhythms during sleep deprivation. Behav Brain Res 103:185-194.

Wyatt JK, Cecco AR, Czeisler CA, and Dijk DJ (1999) Circadian temperature and melatonin rhythms, sleep, and neurobehavioral function in humans living on a 20-h day. Am J Physiol 277:R1152-R1163. 